\title{
Agnes Pless und Kardinal Albrecht von Brandenburg
}

\author{
von Andreas Tacke
}

Luthers Beurteilung, daß die newen Bisschoven allesamt huren wirte seien ${ }^{1}$, werden viele Zeitgenossen geteilt haben und, wie von Luther selbst, auch auf Kardinal Albrecht von Brandenburg (1490-1545) angewandt worden sein. Zu offensichtlich waren die Sitten in den Klöstern und beim Klerus verfallen, als daß sich die Vertreter der Kirche noch große Mühe gegeben hätten, ihre Mätressenwirtschaft zu verbergen. Manchen von ihnen schien dann auch die Meinung Luthers ehrlicher, die Konsequenz zu ziehen und in den Stand der Ehe zu treten. Der Reformator verteidigte immer wieder Männer und Frauen, die das Gelöbnis der Keuschheit aufgaben, gegen die Verfolgung der Altgläubigen. In dieser Angelegenheit empfahl er auch Kardinal Albrecht, daß die Bischoffe zuvor ihre Hurn von sich trieben, ehe sie fromme Eheweiber von ihren Ehemännern scheideten ${ }^{2}$. Da auch der Kardinal den Ruf eines Lebemannes hatte und sich einem verschwenderischen und nur allzu weltlichen Hofleben hingab, war für den Wittenberger der Ratschlag nur folgerichtig, das sich Ewer Churfürstlich gnad in den Ehelichen standt begeben und das Bistumb zu weltlichen furstenthum macheten und den falschen namen und scheyn geystlichs standts fallen und faren lassen ${ }^{3}$. Da entsprechende Belege fehlen, ist es müßig, darüber zu spekulieren, ob Albrecht wirklich ernsthaft einen solchen Schritt, die Bistümer Halberstadt und Magdeburg zu säkularisieren, erwog. Bedauerlich ist, daß wir nur indirekte Nachrichten über diesen Teil seines Lebens erhalten. Die wenigen Quellen stehen im Kontrast zu der Tatsache, daß Albrechts Frauengeschichten landeskundig gewesen sein müssen. Jedoch ist es einmal möglich, den Schleier der Diskretion zu lüften und eine Mätresse des Kardinals urkundlich nachzuweisen. Man hat wohl zu Recht vermutet, daß die Unterlagen, die über Geschenke, Zuweisungen etc. an seine übrigen Geliebten existierten,

\footnotetext{
${ }^{1} \mathrm{Vgl}$. WA 30,2, S. 338.

${ }^{2}$ WA Br. 2, Nr. 442 vom 1.12.1521, S. 408.

${ }^{3}$ WA 18 , S. 408.
} 
vernichtet wurden. Daß sich über die Kardinalsmätresse, die Frankfurter Bürgerstochter Agnes Pless, mehr erhalten hat, ist nur der Tatsache zu verdanken, daß sie nach dem Ableben Albrechts (24. September 1545) in eine heftige Auseinandersetzung gezogen wurde, die die drei Bistümer des Verstorbenen um seine Hinterlassenschaft untereinander ausfochten. Natürlich ging es um Geld, Agnes Pless wird Zeugin und Opfer dieses Streits, dessen Dokumentation über seinen sozial- und sittengeschichtlichen Aspekt hinausweist.

Die Hinterlassenschaft Albrechts, deren Verbleib nicht testamentarisch geregelt war, aufzuteilen, erwies sich als schwierig. Drei Bistümer hatte der am 28. Juni 1490, als jüngster Sohn des brandenburgischen Kurfürsten Johann Cicero geborene Kardinal unter sich vereint. Am 26. Januar 1509, also achtzehnjährig, wurde er Domherr des Erzbistums Magdeburg, vier Jahre später (1513) Erzbischof von Magdeburg und Administrator des Bistums Halberstadt. Bald darauf (1514) wurde er zum Erzbischof von Mainz erwählt und hatte damit die nach Kaiser und König ranghöchste Stellung des Erzkanzlers und Primas des Deutschen Reiches inne. Die Unkosten für das Mainzer Pallium sowie für die Erlaubnis der Ämterhäufung wurden bekanntlich durch den $A b l a ß$ eingetrieben, der für Luthers Niederschrift der 95 Thesen den Ausschlag gab. Am 1. August 1518 wurde Albrecht auf dem Reichstag zu Augsburg im Auftrag Leos X. von Cajetan zum Kardinal erhoben. Damit hatte er eine Machtfülle erreicht, die nicht nur den Zeitgenossen Anlaß zum Staunen gab. Auch aus heutiger Sicht steht diese ekklesiale Karriere für die deutsche Renaissancezeit einzigartig da.

Um so erstaunlicher ist, daß bis jetzt noch keine gründliche, wissenschaftliche Biographie zu diesem Mann vorliegt ${ }^{4}$. Meistens begegnet man der Auffassung, der Kardinal sei nur ein phlegmatischer Gegenspieler Luthers gewesen, den das Schicksal unglücklicherweise in diese Periode des radikalen Wandels gesetzt hätte. Doch ist Vorsicht geboten: häufig machen sich auch heutige Forscher die Sichtweise Luthers bezüglich dieses Kardinals zu eigen. Nur wenige Kilometer trennten damals die Vertreter eines sich polarisierenden Glaubensstreites, Luther in Wittenberg und Kardinal Albrecht in Halle an der Saale. Albrecht wählte sicherlich gerade hier seine Hauptresidenz, um von dem mächtigen Mainzer

${ }^{4}$ Ein Überblick mit Literaturverweisen bei G.A. B e $\mathrm{n}$ r a t h, Albrecht von Mainz, in: TRE 2, 1978, S. 184-187. 
Domstift unabhängig zu sein. $\mathrm{Da} ß$ von Wittenberg die Reformation ausging und auch schnell die Saalestadt erreichte, wurde für ihn folgenschwer. Gerade die Nähe zu Wittenberg brachte ihm eine schmachvolle Niederlage ein, er mußte 1540 Halle verlassen und in das ungeliebte Erzstift Mainz, nach Aschaffenburg ziehen ${ }^{5}$. In der Geschichtsliteratur war man lange geneigt, diesen Tatbestand auf einen phlegmatischen Charakter Albrechts zurückzuführen. Doch keineswegs gab der Kardinal Halle kampflos preis, daher ist ein kurzer Blick auf die dortigen Verhältnisse zu werfen, zumal Agnes Pless für mehr als ein Jahrzehnt mit Albrecht dort verweilen sollte.

In nur zwei Jahrzehnten hatte Albrecht von Brandenburg Halle in eine glanzvolle Renaissancemetropole verwandelt. Die fortschreitende heutige Zerstörung dieser Stadt durch Umweltverschmutzung und die überall um sich greifende Betonplattenbauweise macht schmerzlich bewußt, daß eine kunsthistorische Arbeit über Albrechts Hallenser Mäzenatentum weder vorliegt noch eine Dokumentation für eine spätere Herausgabe angelegt wurde ${ }^{6}$. Vieles wird bald schon verschwunden sein, als ob es nie vorhanden gewesen wäre. Nur weniges läßt dann noch ahnen, daß Halle ein Zentrum der deutschen Renaissancekunst war. Sein Hauptaugenmerk richtete Albrecht auf den Bau der Neuen Residenz, die seine geplante, aber nicht zustandegekommene Universität aufnehmen sollte, das Stift und die Burg, sowie die weiten Parkanlagen am anderen Ufer der Saale. Eine

${ }^{5}$ Siehe zu der Entwicklung W. D e 1 i u s , Die Reformationsgeschichte der Stadt Halle, Berlin 1953, und F. S c h r a d e r, Kardinal Albrecht von Brandenburg, Erzbischof von Magdeburg, im Spannungsfeld zwischen alter und neuer Kirche, in: Von Konstanz nach Trient (...). Festschrift für A. Franzen, hg. von R. Bäumer, München u.a. 1972, S. 419-445.

${ }^{6}$ Bedenklich der Versuch eines allgemeinen Überblicks bei M. v o n R o e s g e n, Kardinal Albrecht von Brandenburg. Ein Renaissancefürst auf dem Mainzer Bischofsthron, Moers 1980. Zu Halle nach wie vor als Standardwerk anzusehen P. R e dl i c h, Cardinal Albrecht von Brandenburg und das Neue Stift zu Halle. 1520-1541 (...), Mainz 1900. Teilaspekte bei U. S te i n m a n n, Der Bilderschmuck der Stiftskirche in Halle. (...), in: Forschung und Berichte, Staatliche Museen zu Berlin (Ost) 11, 1968, S. 69-104; I. L ü h m a n n-S c h mi d, Peter Schro. Ein Mainzer Bildhauer und Backoffen-Schüler, in: Mainzer Zeitschrift 70, 1975, S. 1-62 und Bd. 71-72, 1976/77, S. 57-100; J. R a s $m$ u s s e n, Untersuchungen zum Halleschen Heiltum (...), in: MüJb 27, 1976, S. 59-118 und Bd. 28, 1977, S. 91-132, und A. T a c k e, Quellenfunde und Materialien zu Desideraten der Berliner Kirchengeschichte des 16./17. Jahrhunderts. Mit Anmerkungen zu dem Hallenser Vorbild des Kardinal Albrechts von Brandenburg, in: Berliner Theologische Zeitschrift, Theologia Viatorum N.F. 5, 1988, 2, S. 237ff., bes. 246-248. 
Zielscheibe für polemische Kritiken bot seine Stiftskirche, die auch das Hallische Heiltum, eine über die Landesgrenze hinaus wegen ihres Umfangs und ihrer Pracht bekannten Reliquiensammlung, barg. Vielleicht gehört es zu Albrechts persönlicher Tragik, zu spät oder auch gar nicht verstanden zu haben, daß diese stummen Zeugen des alten Glaubens und deren ständige Vermehrung bei den angesprochenen Landeskindern genau das Gegenteil von dem bewirkte, was er sich von ihnen erhoffte. Statt Festigung der Treue zur Kirche der Väter löste Albrechts Stiftskirche nur Spott und Hohn und den Wunsch nach radikalen Veränderungen aus. Jedoch ist zu konstatiern, daß Albrecht mit seinen Hallenser Unternehmungen eine Antwort auf Luthers Lehre versuchte. Man könnte vielleicht von einer Gegenreformation ante litteram sprechen. Die Zeichen der Zeit waren allerdings anders zu deuten, als es Albrecht tat. Doch keinesfalls ist daraus der Schluß zu ziehen, er sei ein willensschwacher Gegner des Wittenbergers gewesen. Vielmehr beherrschte er das ganze Repertoire meisterlich, welches seine Vorgänger noch mit Erfolg anwenden konnten, bei ihm jedoch als antiquiert aufgenommen wurde. Es ist nicht ohne Ironie, daß Albrecht für die wichtigsten malerischen Arbeiten im Stift Lucas Cranach d.Ä. gewinnen konnte. Aus seiner Werkstatt sollte auch der spätere Hofmaler des Kardinals kommen, nämlich Simon Franck ${ }^{?}$.

Wann Agnes Pless die Mätresse des Kardinals wurde, verschweigen die Quellen. Eine nach wie vor in Halle lebendige mündliche Überlieferung läßt sie die Nachfolgerin der legendären Ursula Riedinger werden, über die es zwar keine schriftlichen Nachrichten gibt, aber deren Tod ungefähr in die Zeit um 1526-28 fällt. Damit begegnet Agnes Albrecht in den Jahren, in denen sich der Kardinal auf dem Höhepunkt seiner Macht befand. Agnes Pless ${ }^{8}$ (geb. um 1502) war eine geborene Strauss, Tochter des Hans Strauss, Metzger zu Frankfurt am Main, und Ottilia Strauss, geb.

${ }^{7} \mathrm{Zu}$ der Ausstattung der Stiftskirche mit einem Heiligen- und Passionszyklus (um 1520-25) durch Lucas Cranach d. Ä. und Simon Franck siehe demnächst den Druck meiner Dissertation: Zwei katholische Hauptwerke von Lucas Cranach d.Ä. Fallstudien zur konfessionellen Ungebundenheit des Künstlers in der deutschen Reformationszeit.

${ }^{8}$ Die folgenden Daten sind entnommen aus A.F. W ol fert, Heraldische Spuren der Verwandtschaft des Kardinals Albrecht von Brandenburg in Aschaffenburg, in: Aschaffenburger Jahrbuch für Geschichte, Landeskunde und Kunst des Untermaingebietes 6, 1979, S. 127-136. Vgl. (mit einigen Irrtümern) W.K. Z ü $1 \mathrm{c} \mathrm{h}$, Kardinal Albrecht von Brandenburg und Agnes Strauß-PleB. Die Geschichte einer romantischen Liebe und ihres tragischen Ausgangs, in: Hallische Nachrichten, Dienstag 2.4.1929, Jg. 41 Nr. 76, S. 13. 
Semer. Kenntnis erhalten wir von einem Bruder Johann Strauss, der 1546 Schultheiß zu Auheim ist und 1556 Bürger zu Hanau. 1521 heiratete Agnes den Frankfurter Bürger Hans Pless, der vor 1527/28 verstarb, und dessen Namen sie später beibehielt. Wie Albrecht sie kennenlernte, muß dahingestellt bleiben. Sie wird den Kardinal, der wegen der drei Bistümer oft unterwegs war, häufig begleitet haben. Doch scheint ihr Hauptaufenthaltsort ebenfalls in Halle gewesen zu sein. Dort besaß sie nämlich nach eigenen Aussagen (siehe Anhang) ein Haus, zum „Grunen hofe" genannt. Ein Haus zum „Grünen Dill (Hof)" ist in der Großen Klausstraße nachgewiesen worden ${ }^{9}$. Die Große Klausstraße verband das Klaustor (und der dazugehörenden Brücke über die Saale) mit dem Markt. Sie existiert heute noch und liegt interessanterweise ganz in der Nähe der Neuen Residenz. Es ist zu vermuten, daß Agnes ihr Haus durch ihre Stellung als Kardinalsmätresse erwerben konnte. Das hier im Anhang abgedruckte Dokument zeigt unmißverständlich, daß sie durch ihre Beziehung zum Kardinal eine sehr vermögende Frau wurde, ihre Hofhaltung der einer Fürstin gleichkam. Wie die Hallenser Jahre im einzelnen verliefen, bleibt im Dunkeln. Vielleicht wohnte ebenfalls in Halle ihre Mutter Ottilia Strauss, denn diese war später in Aschaffenburg bei ihr.

Es ist durchaus möglich, daß Agnes' Porträt auf einem der vielen Gemälde zu sehen ist, die Albrecht von Brandenburg bei den berühmtesten Malern seiner Zeit anfertigen ließ. Die meisten Bilder haben sich von Lucas Cranach d.Ä. und seiner Werkstatt erhalten. Vor allem für Albrechts Neues Stift fertigte Cranach mit seinem Meisterschüler Simon Franck über hundert, oft lebensgroße Heiligendarstellungen an. Sehr häufig verlieh der Kardinal sein Porträt den Heiligen, sein Lieblingsheiliger war Erasmus, von dem sich mehrere Bilder erhalten haben, auf denen Albrecht als Heiliger Erasmus dargestellt ist. Simon Franck zeigt in seinen Gemälden eine große Vorliebe für renaissancehafte Formen. Kostbare Stoffe und Schmuck entsprechen bei den Heiligen der zeitgenössischen Tracht. Vergleicht man diese Darstellungen mit den Angaben, die Agnes Pless von ihrem Besitz an Ketten, Ringen und Kleidern macht (siehe Anhang), so wird verständlich, daß eine Überlieferung in Halle besagt, daß ihre Vorgängerin Ursula Riedinger, die als Kardinalsmätresse sicherlich ebenso

${ }^{9}$ Ein solches Haus ist 1425 (Druckfehler, richtig 1525?) mit dem Besitzer „Hans Hake" nachgewiesen, siehe S. v on S c h u l t z e-G allér a, Topographie oder Häuser $=$ und Straßen=Geschichte der Stadt Halle a.d. Saale, Bd. 1, Halle 1920. 
reich war wie Agnes, sehr häufig auf Gemälden als Heilige Ursula zu sehen sei. Die Heilige Ursula war eine Hauptpatronin des Stifts.

Mit Albrecht verließ Agnes Pless Halle endgültig am Anfang des fünften Jahrzehnts des 16. Jahrhunderts. Die Stadt war lutherisch geworden, für die „Trutzburg Roms“ gab es kein Halten mehr. Vorausgegangen war ein für Albrecht demütigender Machtverfall. Immer weniger konnte er dem lutherisch gesinnten Hallenser Bürgertum etwas entgegensetzen, mußte er mit dem Rat der Stadt für ihn unvorteilhafte Kompromisse aushandeln. Wortgewaltig verspottete Luther von Wittenberg aus den Kardinal, der in Halle auf verlorenem Posten für das Papsttum stand. Agnes Pless folgte dem Kardinal nach Aschaffenburg. Dort wird sich Albrecht bis zu seinem Tod vorwiegend aufhalten, unübersehbar auch dabei die deutliche räumliche Distanz zum Mainzer Domstift. Der Kardinal hatte nach seinem Rückzug aus Halle 1540 sein Testament ${ }^{10}$ gemacht und dabei Aschaffenburg großzügig bedacht. Neben dem dortigen Stift und der Beginenkirche war es vor allem seine Residenz, das Aschaffenburger Schloß, welches einen großen Teil der Hallenser Kunstwerke erhielt. Für den Transport und die erneute Unterbringung war sein Hofmaler Simon Franck verantwortlich. Er hatte schon in Halle, neben seiner Tätigkeit als Maler, organisatorische Aufgaben übernommen. Das Aschaffenburger Schloß brannte 1552 aus ${ }^{11}$, verloren ging damit auch ein großer Teil der Hallenser Kunstwerke.

Agnes Pless erwirbt am 13. Juli 1542 unweit des Aschaffenburger Schlosses ein Grundstück in der clein Weber gassen gelegen, welches zuvor die behausu(n)g des ... hern Dietherichs von Lauthern Doctor etc. ... (vor jaren des Monchs hauss genandt) gewesen war. Wo sich Agnes in den zwei Jahren zwischen 1540, dem Weggang von Halle, und 1542, der Erwähnung in Aschaffenburg, aufhielt, entzieht sich unserer Kenntnis. In dem Vertrag ${ }^{12}$ mit dem Dechant vnd Capittel sanct Peter vnd Alexanders Stiefts Kirchen zu Aschaffenburgk werden die Einzelheiten festgehalten: Derweil aber solche Zinss hauss vnd flecken nhümer abgebrochen vnd in einen baw daselbst khomen vnd verbaut worden ... so sindt wir von wegen

${ }^{10}$ StA Würzburg, Mz. Urk. Libell 37; vgl. den Abdruck bei R e dl i c h, Neues Stift (wie Anm. 6), Beilage 37.

${ }^{11}$ Siehe O. S chulze-Kolbitz, Das Schloß zu Aschaffenburg (= Studien zur Deutschen Kunstgeschichte 65), Strassburg 1905, S. 16.

${ }^{12}$ StiA Aschaffenburg, Liber Camerae V., B1. 209r-209v, 13. Juli 1542. 
der Erbaren vnd tugenthafften frawen Agnesen Blessen inhabern gedachter Zinss fleck(en) freuntlich ersucht vnd gebeten word(en), wir wolt(en) die gepurliche ablosung gestatten. Es ist zu vermuten, daß das Aschaffenburger Stift $^{13}$ auf Druck des Kardinals dieses Grundstück abgab. Denn zwei Jahre zuvor (28.10.1540) hatte das Stift, ebenfalls bey dem Schloss gelegen, ein Haus dem Hofmaler Albrechts, Simon Franck, und seiner Frau Anna zu für das Stift ungünstigen Konditionen überlassen müssen ${ }^{14}$. Somit waren nun unmittelbar bei der Residenz Albrechts treue, jedoch ungleiche Gefolgsleute aus Halle untergekommen. Agnes Pless lie $B$ die Gebäude auf dem Grundstück abreißen und durch einen Neubau ersetzen. Dazu verwendete sie unter anderem den Erlös von 2200 Gulden, welchen sie aus dem Hausverkauf in Halle erzielte (s.u.). Ihre Vermögensverhältnisse waren im übrigen $\mathrm{so}, \mathrm{da} ß$ sie wahrscheinlich einen sehr aufwendigen Neubau errichten lassen konnte. Nach Albrechts Tod wird sie beteuern, daß dafür nur eigene und nicht Gelder des Kardinals verwendet wurden.

Zumindest in Aschaffenburg, vielleicht auch in Halle, wurde Agnes von ihrer Mutter Ottilia Strauss, geb. Semer, begleitet, denn diese verstarb 1543 am Di(en)stag nach Vrbani (nach dem 25. Mai) dort. Ihr Epitaph befindet sich nach wie vor im Aschaffenburger Stift St. Peter und Alexander. Alfred A. Wolfert hat erst jüngst den Zusammenhang mit Albrecht hergestellt ${ }^{15}$. Das Epitaph zeigt die Verstorbene kniend vor einer Darstellung mit Christus als Schmerzensmann sowie Maria und Johannes. Neben einer Inschrifttafel findet sich das Wappen der Frankfurter Bürgerfamilie Strauss (in Rot ein natürlicher Vogel Strauß mit blauem Hufeisen im Schnabel) und das der Familie Semer.

Aus dem im Zweiten Weltkrieg im Frankfurter Stadtarchiv verbrannten Testament der Agnes Pless ${ }^{16}$, zu dem der Grünewaldforscher Walter K.

${ }^{13}$ Siehe zum Stift F. M a d e r, Die Kunstdenkmäler des Königreichs Bayern, UnterFranken Bd. 3 Heft 19: Stadt Aschaffenburg, München 1918, S. 25-152 (mit älterer Literatur); M. K l e w i t z, Die Baugeschichte der Stiftskirche St. Peter und Alexander zu Aschaffenburg (= Veröffentlichungen des Geschichts- und Kunstvereins Aschaffenburg), Aschaffenburg 1953, und das Urkundenbuch des Stifts, bearb. und hg. von M. Th i e l, Bd. 1-, Aschaffenburg 1986-.

${ }^{14}$ StiA Aschaffenburg, U 2579, Pergamenturkunde mit dem Siegel Simon Francks.

${ }^{15} \mathrm{Vgl}$. W olf e r t, Spuren (wie Anm. 8), S. $135 \mathrm{f}$.

16 Freundliche Mitteilung von Bernhard Reichel (Stadtarchiv Frankfurt/Main, 22.4.1988). Dazu gehörten die ebenfalls verbrannten Inventare 1547 Nr. 33 und 1565 Nr. 34. 
Zülch einige Angaben lieferte ${ }^{17}$, ging hervor, daß Albrecht Agnes Pless zur Vorsteherin (Mutter) der Beginen in Aschaffenburg machte. Die kleine Niederlassung der Beginen lag im Tiergarten, unmittelbar an der Aschaffenburger Stadtmauer ${ }^{18}$. Sie ist heute als "romantische Ruine“ in den Stadtpark Schöntal eingebunden. Gestiftet wurde die Niederlassung von der Familie derer von Gonsrode, die Bestätigung erfolgte am 20. Dezember 1527 durch Albrecht. Das besondere Interesse des Kardinals an dieser kleinen, nur sechs Schwestern umfassenden Niederlassung, ist daraus zu ersehen, daß er im Jahre 1528 für die Beginenkirche zum Heiligen Grab fünf Altäre dotierte. Vielleicht wurde der Hauptaltar dieser Kirche von Matthias Grünewald, dem Künstler des Isenheimer Altars, gemalt. Nach seinem Rückzug aus Halle wandte sich der Brandenburger erneut der Heiliggrabkirche zu. Verschiedene Um- und Anbauten tragen sein Wappen und die Jahreszahlen 1543 bzw. 1544. Simon Franck forderte für die Betreuung dieser Arbeiten nach Albrechts Ableben vom Mainzer Domkapitel den noch ausstehenden Lohn ein. Seine Aufgabe war es auch, einige der Hallenser Kunstwerke hier neu aufzustellen. Dazu zählten vor allem Altäre und Gemälde, die durch Albrechts Wappen und sein Porträt in einer engen Beziehung zum Brandenburger standen. Aber auch kostbare Kleinodien, Reliquiare und kirchliches Gerät, wie auch Antependien und Ornata, gehörten dazu. Agnes Pless mußte nach Albrechts Tod über die Zuwendungen an Cleinoth vnd Ornata, die die Niederlassung der Beginen vom Kardinal erhalten hatte, Auskunft geben. In ihre Zeit als Beginenmutter fällt auch ein sehr privates Geschenk, welches Albrecht zum Neujahr von ihr erhielt, ein Bierkrug: 1 silbern zum teil vorgulte bierkanden, welche mein gnedigster her stetz gebrauchtt; diese kanden wart im zum newen jhar geschanckt zu Aschaffenburgk ym dhirgarthen ${ }^{19}$. Man kann davon ausgehen, daß Agnes nicht ihre Zeit, wie es bei den ihr anvertrauten "Schwestern" der Fall war, mit der Pflege von Kranken, Alten und Siechen ausfüllte, oder Tage und Nächte klagend und betend

${ }^{17}$ W.K. Z ul $1 \mathrm{c} \mathrm{h}$, Der historische Grünewald. Mathis Gothardt=Neithardt, München 1938, S. 400ff. Die Quellenangaben und ihre Auswertung sind bei Zülch oft flüchtig und in manchen Fällen affirmativ eingesetzt, Vorsicht ist geboten.

${ }^{18}$ Dazu demnächst meinen Aufsatz: Die Heiliggrabkirche der Beginen bei Aschaffenburg. Mutmaßungen zu einer Memorialkirche Kardinal Albrechts von Brandenburg mit dem Spätwerk des Matthias Grünewald.

${ }^{19}$ Redlich, Neues Stift (wie Anm. 6), 204*f., zitiert wird aus einer Aufzählung der Hinterlassenschaft Albrechts von 1545. 
am Grab Christi während der Osterliturgie verbrachte ${ }^{20}$. Sicherlich werden andere Beweggründe als die der mildtätigen Nächstenliebe den Kardinal geleitet haben, als er sie zur Mutter der Beginen machte, vielleicht, um Agnes auch nach seinem Tod versorgt zu wissen. Doch eng mit dem Schicksal Albrechts verbunden, überdauerte die Heiliggrabkirche nicht lange sein Ableben. Sofort nach seinem Tod wurde sie durch das Aschaffenburger Stift ausgeräumt. Albrecht hatte dem Stift St. Peter und Alexander die Kunstwerke dieser Kirche als Pfand angegeben, für den Fall, daß er die Zurückzahlung eines Kredites schuldig blieb ${ }^{21}$. Das Stift hatte zu Recht eine Sicherheit verlangt, denn Albrecht blieb wie so oft ein säumiger Zahler. Bereits in der Mitte des 16. Jahrhunderts war der Komplex in ruinösem Zustand, eine Folge von Brandschatzungen während des Schmalkaldener und des Markgräfler Krieges.

Am 24. September 1545 verstarb Kardinal Albrecht von Brandenburg in der Mainzer Moritzburg ${ }^{22}$, erst fünfundfünzigjährig. Viele werden nicht unglücklich gewesen sein, denn Albrecht hatte immer mehr von ihm Abhängige in seine zweifelhaften Geldunternehmungen gezogen. Auch das mächtige Mainzer Domkapitel konnte sich nicht den Geldforderungen entziehen. Doch schwer krank mußte Albrecht in den letzten Monaten oft erniedrigende Domkapitelsitzungen hinnehmen. Das Kapitel leistete es sich am Ende seines Lebens, immer offener harte Bedingungen für neue Darlehen zu diktieren und rechnete dem Kardinal des öfteren vor, daß das neu aufgenommene Geld nicht einmal reichen würde, um die ausstehenden Zinsen für alte Kredite zu tilgen, so daß er das neu gewonnene Geld nicht einmal für den angegebenen Zweck verwenden könnte ${ }^{23}$. Albrecht wird geahnt haben, daß seine Günstlinge es nach seinem Tode schwer

${ }^{20}$ Siehe E.G. N e u m a n n, Rheinisches Beginen- und Begardenwesen. Ein Mainzer Beitrag zur religiösen Bewegung am Rhein, (Diss. phil. Mainz 1959) (= Mainzer Abhandlungen zur mittelalterlichen und neueren Geschichte 4), Meisenheim a. Glan 1960, zur Aschaffenburger Niederlassung bes. S. 69, S. 84, S. 88, S. 98 f. und S. 126.

${ }^{21}$ StiA A schaffenburg, Liber Camerae V., B1. 224v-225v, und U 4127. Der Abdruck demnächst in meinem Aufsatz (wie Anm. 18).

${ }^{22}$ Zur Burg siehe U. Z a h 1 e r, Das Kurfürstliche Schloß zu Mainz. Studien zur Bauund Stilgeschichte (= Saarbrücker Hochschulschriften, Kunstgeschichte 8), (Diss. phil.) St. Ingbert 1988, S. $15 \mathrm{ff}$.

${ }^{23}$ Vgl. die Protokolle der letzten Sitzungen bei F. H e r r m a n n, Die Protokolle des Mainzer Domkapitels. 1514-45, 2 Bde., Paderborn 1930-32. Zur Verschuldung vgl. auch J. M a y , Der Kurfürst, Cardinal und Erzbischof Albrecht II. (...), 2 Bde., München 1865-75; hier Bd. 2, S. $475 \mathrm{ff}$. 
haben würden. Am gefährdetsten schien ihm vor allem seine Mätresse, denn kurz vor seinem Ableben läßt er seinen langjährigen Sekretär Melchior Vogt schwören, Agnes Pless nach seinem Tod zu beschützen. Dieser wird jedoch den Eid nicht halten, was sich bei Albrechts Nachfolger karrierefördernd auswirkte, denn unter dem Erzbischof Sebastian von Heusenstamm (1545-1555) stieg er in die Stelle eines Rates auf. Agnes Pless, die noch in den letzten Stunden bei Albrecht auf der Mainzer Moritzburg weilte, wurde sofort nach der Bekanntwerdung seines Todes auf Anordnung des Domkapitels verhaftet und bis etwa Mitte November, also circa zwei Monate gefangengehalten. Dabei spielten keineswegs sittliche Gründe eine Rolle. Es ging dem Kapitel vielmehr um Geld, um sehr viel Geld. Alles was Agnes in ihrem Aschaffenburger Haus verwahrte und sie in Mainz bei sich hatte, wurde vom Kapitel umgehend ,beschlagnahmt ${ }^{6} . \mathrm{Zu}$ vermuten ist, daß die Mainzer Domherren diese Angelegenheit am liebsten in aller Stille geregelt hätten. Doch meldeten Vertreter der Bistümer Halberstadt und Magdeburg ihre Rechte auf einen Teil der Hinterlassenschaft Albrechts an, und dazu zählten auch sie ebenfalls den Besitz von Agnes Pless.

Am Montag nach Epiphanie domini (nach dem 6. Januar) ... im sechsundviertzigsden Jahr, also ungefähr dreieinhalb Monate nach Albrechts Tod, kamen die geschickten aus Halberstadt und Magdeburg vmb dreij iehr nach Mittage in Aschaffenburg an. Ihrem ausführlichen Bericht ${ }^{24}$ ist zu entnehmen, daß sie wenig ausrichten konnten. Der neue Mainzer Erzbischof, Sebastian von Heusenstamm, zeigte sich kaum kooperativ. Ja, man verweigerte ihnen sogar vorläufig den Einblick in Albrechts Testament, geschweige, daß man ihrer Bitte nachkam und ihnen eine Abschrift davon aushändigte. Deshalb versuchten sie, so gut wie möglich sich selbst eine Meinung darüber zu bilden, was in ihre Bistümer gehörte. Am einfachsten waren die Besitzverhältnisse zu klären, wenn die Gegenstände mit den Wappen der Bistümer geziert waren. So hielten sie auch eifersüchtig in einer Auflistung der Hinterlassenschaften Albrechts fest, daß Symon Mahler, also Simon Franck, an einem Wappen Reparaturen vorgenommen hatte ${ }^{25}$ und vermuteten, daß vielleicht durch solche Arbeiten Manipulatio-

${ }^{24}$ StA Magdeburg, Rep. A 2 Nr. 20, Bl. 14r-21v; eine Zusammenfassung und Auszüge bei R edli c h, Neues Stift (wie Anm. 6), S. 241*-245*.

${ }^{25} \mathrm{Vgl}$. R e d l i c h, Neues Stift (wie Anm. 6), S. 208*. Ein Teilinventar der Gesandten über Aschaffenburger „Sijlbergeschir" hat sich erhalten im StA Magdeburg, Rep. A 2 Nr. 20, Bl. 11r-12r, vgl. dazu auch R e dl i c h, Neues Stift (wie Anm. 6), S. 240*f. 
nen der Wappen zu ihren Ungunsten geschehen seien: das Meinzische wapen an Magdeburgisch oder Halberstadisch wapen stadt gesezt worden war (s.u.). Als sie davon Kenntnis erhielten, daß der neue Erzbischof die frühere Mätresse Albrechts sehr schlecht behandelt haben sollte, nahmen sie Kontakt zu Agnes Pless auf. Ihre Beweggründe waren allerdings nicht von Mitleid geleitet. Penibel hielten sie in einem Protokoll alles fest, was Agnes in dem Gespräch als ihren vom Mainzer Kapitel entwendeten Besitz angab und forderten diesen nun ihrerseits vom Mainzer Erzbischof für ihre Bistümer zurück: Darneben auch gebetten, Nachdem Seine Churf(ürstliche) g(naden) (Albrecht von Brandenburg) der Agnes blessin vill stattlichs an parschafft, Ringen, gulden Kethen, Cleinothen, Silbergeschirre, Cleidern vnnd anderm im Erzstifft Magdenburgk gegeben, welichs alles in den Stifften Magdenburgk vnd Halberstat erzeuget vnnd denselbigen zuegehoris. Vnnd izo von einem hochwirdigen Thumbcapittell zu Meinz in vorwarung genohmen vnd inventiret. Das sein Churf. g. (Sebastian von Heusenstamm) bei dem Capittell gnediglich verschaffen wolle, das solichs den geschickten widderumb zuegestelt werdenn moch$t e^{26}$. Um ihre Forderungen detailliert nachweisen zu können, wurden Agnes Aussagen schriftlich festgehalten: Vngefarlicher Bericht soviel Agnes Plessin muntlich angezeigt vnd Doctor Barthen vnd Johan Herman bericht ${ }^{27}$. Er hat einen Umfang von neun Seiten und ist nicht nur interessant wegen der Angaben zu den Ereignissen nach Albrechts Tod und zu den Vermögensverhältnissen Agnes, sondern hält auch Vorgänge im Zusammenhang mit Albrecht fest, von denen man bis jetzt keine Kenntnis hatte.

Es drängt sich beim Lesen das Gefühl auf, daß die beiden Protokollanten Doctor Barthen vnd Johan Herman bei Agnes den Eindruck erweckten, sie seien auf ihrer Seite, um von ihr um so bereitwilliger Auskunft zu erhalten. Ziemlich am Anfang des Berichts $\left(25^{r}\right)$ vermerkten sie, und das kann man doch nur als Taktik verstehen, angesichts der Tatsache, daß die geschickten Agnes Besitz für ihre Bistümer fordern werden, daß Agnes für die ihr vom Mainzer Kapitel zugefügten Schmerzen 100 Floren als

${ }^{26}$ StA Magdeburg, Rep. A 2 Nr. 20, B1. 14v.

${ }^{27}$ StA Magdeburg, Rep. A 2 Nr. 20, B1. 25r-29r. Beim Abdruck wurden Zahlenangaben aufgelöst und in der arabischen Schreibweise wiedergegeben. Um die Anmerkungen nicht unnötig zu belasten, wurden die Nachweise von Personen, Ortsangaben etc. direkt in Klammern im Text vorgenommen. 
Entschädigung erhalten soll. Ihr war durch das Domkapitel übel mitgespielt worden, sodaß sie nur allzu gern geglaubt haben mochte, in den Vertretern aus Magdeburg und Halberstadt Verbündete gefunden zu haben. Rechtlich kompliziert wurde ihre Situation dadurch, daß sie dem Mainzer Kapitel unterschreiben mußte, nichts gegen das ihr zugefügte Leid zu unternehmen. Dafür wurden fünf Männer als Bürgen eingesetzt $\left(26^{v}\right)$. So bittet sie in dem Bericht mehrmals den neuen Erzbischof von Magdeburg (1545-1550), Johann Albrecht von Brandenburg (14991550), wenn er etwas gegen das ihr zugefügte Unrecht unternehmen wolle, den Eindruck zu vermeiden, sie hätte um seine Hilfe nachgesucht. Kardinal Albrecht selbst hatte ihr empfohlen, wie sie angibt, nach seinem Tod Beistand von seinem Koadjutor (dem späteren Erzbischof) von Magdeburg Johann Albrecht und von dem Markgrafen Albrecht von Brandenburg-Anspach (1490-1568) zu fordern. Vielleicht erhoffte sie sich auch Hilfe von Herzog Heinrich von Braunschweig-Wolfenbüttel (1489-1568), der ihr einst eine kostbare Kette geschenkt hatte $\left(27^{v}\right)$.

Am wichtigsten war den Gesandten wohl der Teil des Berichts, in dem Agnes eine Aufzählung des vom Mainzer Kapitel in Aschaffenburg und Mainz beschlagnahmten Besitzes gab ( $26^{x} \mathrm{f}$.). An silbernem und goldenem Geschirr alleine 400 Mark (zu je ca. 233 Gramm), 15 Pfund an Goldketten, hinzu kamen 90 kostbare Gürtel, der wertvollste für 1200 Gulden. Neben den 32 Röcken war der Rock aus Perlen vom Seidensticker Amanden wohl der wertvollste. Aber auch kostbare unbearbeitete Seidenstoffe waren darunter, ebenfalls vngefarlich 300 Ringe mit Edelsteinen. Neben allgemeinem Hausrat gehörte Eingemachtes und Wein dazu. Schmerzlich war der Verlust von brieve vnd siegel vber ijhre Zinse vnd Renthe die sie in Halle, Leipzig, Nürnberg und Frankfurt hinterlegt hatte.

Interessant sind auch die Ausführungen zum Kardinal selbst. So vermachte er der Aschaffenburger Beginenkirche Cleinoth vnd Ornata für circa 8000 Gulden, dem dortigen Stift Gegenstände im Wert von 18000 Gulden. Auskunft gab Agnes über eine Münzsammlung und kostbare Teppiche. Sie muß auch Kenntnisse über Albrechts Besuche von Reichstagen gehabt haben, denn sie berichtete (leider zu diesem Punkt nur sehr allgemein), was dort gescheen und an Kostbarkeiten zur Finanzierung der Reichstage vorkaufft vnd auffgangen war. Hatte sie ihn dorthin begleitet? Vom Landtag in Kalbe im Frühjahr 1541 erzählt sie, daß sie selbst Albrecht 1000 Taler vorgestreckt habe, auf deren Rückzahlung sie nicht sonderlich gedrungen hätte. Auf dem Landtag zu Kalbe hatten die Land- 
stände des Erzstifts Magdeburg dem Kardinal die ungeheure Schuldenlast von 536000 Floren abgenommen ${ }^{28}$. Wie weit sie dafür Zugeständnisse auf religiösem Gebiet erreichten, entzieht sich unserer Kenntnis. Das Mainzer Kapitel habe, so weiß Agnes zu berichten, die Annahme von Albrechts Testament (um 1540) davon abhängig gemacht, daß der Kardinal seine Schulden bei ihnen beglich. Vom Kaiser Karl V. (1519-1556) soll der Kardinal jährlich 7000 Taler Pension erhalten haben, bis jetzt ging man immer von 10000 aus.

Der Bericht endet mit der demütigen Bitte Agnes Pless, ihre Offenheit zu honorieren. Die sicherlich zu Lebzeiten Albrechts von Brandenburg einflußreiche Mätresse war jedoch ein Opfer ihrer Beziehung zum Kardinal geworden. Ihr bereitwillig gegebener Bericht und ihr demütiges Bitten nutzten nicht viel, die Bistümer Magdeburg und Halberstadt erhoben nun ihrerseits Anspruch auf die Agnes gehörenden - und vom Mainzer Kapitel beschlagnahmten Besitzungen. Inwieweit die drei streitenden Bistümer in dieser Angelegenheit eine Einigung erzielten, ist nicht überliefert.

Agnes Testament (vom 16. Mai 1546) läßt aber vermuten, daß die Bistümer nicht aller Besitzungen der ehemaligen Kardinalsmätresse habhaft werden konnten. Denn Agnes Pless starb als sehr reiche Frau, trotz der gar nicht christlichen Plünderungen. Walter K. Zülch faßte seinen Eindruck vom Testament zusammen: „Die einzeln aufgeführte Garderobe ist an Kostbarkeit der Stoffe und Zahl die einer großen Fürstin! An ihrem fabelhaften Goldschmuck befindet sich in Gemmen geschnitten und geprägt überall des Erzbischof Albrechts Bildnis, an Ketten, Ringen und Armschmuck. An einer Goldkette hängt, des Bischofs von Mainz seligen Tochter Anna ${ }^{29}$ Bildnis ““30. Als Testamentsvollstrecker soll Agnes, kurz vor ihrem Tod lutherisch geworden (!), den Landgrafen Philipp I. von Hessen (1509-1567) eingesetzt haben. „Sie interessiert ihn zugleich durch die Zuwendung eines Legates von 4000 Gulden für die hessischen Spitäler. So greift der Agnes Pless Testament hinein in die große Politik jener Tage" ${ }^{\text {"31 }}$. Ihr Ziel war dabei (nach Zülch), daß Philipp von Hessen dem

\footnotetext{
${ }^{28}$ Vgl. W. D e li u s, Kardinal Albrecht und die Wiedervereinigung der beiden Kirchen, in: ZKG 62, 1943/44, S. 187.

${ }^{29} \mathrm{Zu}$ dieser siehe R. H u e n i c k e n, Nachkommen Kardinal Albrechts von Brandenburg, in: Festschrift für Geheimrat Freydank zu seinem 90. Geburtstag (= Ekkehardbücherreihe 9), Halle 1941, S. 23-26.

${ }^{30} \mathrm{Z}$ ä l c h, Grünewald (wie Anm. 17), S. 403.

${ }^{31} \mathrm{Z}$ ül c h, Grünewald (wie Anm. 17), S. 403.
} 
Mainzer Kapitel wieder ihr Eigentum „abjagen“ sollte. Ihren Besitz soll sie nach Zülchs Angaben in Frankfurt deponiert haben, als sie die Stadt verlie $B$ und in Rommershausen den hessischen Adeligen Raban von Holzheim am 17. Februar 1547 heiratete. Zu vermuten ist, daß sie vorher ihrer Aufgaben als Mutter der Beginen in Aschaffenburg entledigt wurde, da die Beginenniederlassung, nach den Plünderungen durch das Stift St. Peter und Alexander, die dem Mainzer Domkapitel zuvorgekommen waren, aufgelöst wurde. Offen muß bleiben, wie lange sie noch Eigentümerin des Aschaffenburger Hauses in der Klein Webergasse geblieben war, welches ja einen nicht unerheblichen Immobilienbesitz darstellte.

Sie erkrankte auf ihrer Hochzeit und verstarb am 4. März 1547, also circa fünfundvierzigjährig. Dies geht aus einer im Frankfurter Stadtarchiv verwahrten Prozeßakte hervor: Zivilprozessakte 1548 Nr. 108/H Rab von Holtzheim contra Lorentzen Villani ${ }^{32}$. Raban von Holzheim klagte auf Herausgabe der beim Kaufmann Lorenz Villani (1491-1559) hinterlegten Vermögenswerte von Agnes Pless. Villani, dem Agnes einen Teil ihres Besitzes anvertraute, war auch schon für Albrecht von Brandenburg tätig gewesen. Als Vertreter der Florentiner Seidenhandlung Petrus Saliti in Frankfurt (dort erwähnt von 1530-1559) wird so mancher kostbare Stoff und vielleicht auch Kunstwerke aus Italien durch ihn an den Kardinal gelangt sein. Ein Porträt des Kaufmanns ist von der Hand Conrad Fabers von Creuznach auf uns gekommen ${ }^{33}$. Der Prozess beschäftigte auch den Rat der Stadt Frankfurt und taucht in den Ratsprotokollen ${ }^{34}$ wie auch im Bürgermeisterbuch ${ }^{35}$ auf. Er zog sich mehrere Jahre hin.

\footnotetext{
${ }^{32}$ StdA Frankfurt/Main, Zivilprozeßakte Nr. 108/H Fasz. 1 und 2 von 1548, Nr. 108/H Fasz. 3 und 4 von 1549.

${ }^{33} \mathrm{Vgl}$. W. B r ü c k e r, Conrad Faber von Creuznach (= Schriften des Historischen Museums Frankfurt am Main 11), Frankfurt/Main 1963, Nr. 13, und A. D i e tz, Frankfurter Handelsgeschichte, Bd. 1, Frankfurt/Main 1910, S. 248, S. 293 f.

${ }^{34}$ StdA Frankfurt/Main, Ratsprotokolle 1548, Bl. 27r (26.4.) und Bl. 53r (7.8.); im Jahr 1549, Bl. 33r (4.7.), Bl. 36r (9.7.) und Bl. 70r (12.11.).

${ }^{35}$ StdA Frankfurt/Main, Bürgermeisterbuch 1548 (die Aufzeichnungen fangen erst mit Donnerstag, dem 3. Mai 1548 an), Bl. 52v (7.8.). Im Jahr 1549, Bl. 24v (4.7.) und B1. $28 v$ (9.7.).
} 
Bericht der Agnes Pless über ihr Schicksal nach Albrechts Tod und über ihre Vermögensverhältnisse

$\left(25^{r}\right)$ Vngefarlicher Bericht soviel Agnes Plessin muntlich angezeigt vnd Doctor Barthen vnd Johan Herman bericht.

Bericht wie sie nach absterben vnsers gnadigsten hern des Cardinals (Albrecht von Brandenburg) etc. seliger vnd loblicher gedechtnuss des abents alsbalde hab von (der Mainzer) sanct Martensburg in ijhr behausung gehen wollen, wie sein Churf(ürstliche) g(naden) ijhr solchs auch zeitlich zuvor also bevohlen. Das Capittel hab sie aber alsbalt zu den gemachen bleijben heijsszen, vorwarn vnd folgent vber etliche tage zu ein sondere stube sambt zweijen Megden bringen, darijnne vorwahrn vnd ein zeitlangck durch eijnen Hescher speijsen ijhr auch ijzo durch diess, dan durch ein andern allerleij bedrowung sie zu eijn Closter zuvorstossen, thun lassen, sie solte dem Capittel alles das ihre zustellen, sie wurden sie wol widerumb versorgen.

Vnnd wiewol Melchior Voigt (erzbischöflicher Sekretär), Vnserm gnedigsten hern beij zeit seins lebens, auff gnedigs gescheenes begern gelobt vnd zugesagt sie nach seiner Churf. g. tode getrewlich vertheijdingen vnd schutzen zuhelffen vnd das beste beij ihr zuthun. So seij er doch der vornembste gewest, der am meisten auff sie gedrungen vnd ihr zugesazt. Nota die schmerz vor $100 \mathrm{fl}$ (oren).

Er hab ihr auch einsmals gesagt, das dije gemeijne burger zu Meinz harte auff sie vorbijttert $\left(25^{v}\right)$ vnd hetten gegen dem Capittel schriefftlich vber sie geclagt. Das doch in der warheijt also nicht gewest. Wie ihr der her Thumsenger solchs selbst angezeigt, das ehr auch des vom Capittel keijnen bevelch gehabt.

Vnnd in sum(m)a sie seij durch Melchior (,) den Burggraven zu Meinz (seit dem 22. Februar 1543 Hans von Aulnbach) vnd andere also bedrowet vnd geengstiget, das sie hat gelubde vnd Burgschafft thun mussen, das ihenig was sie wider das Capittel zusuchen habenn mochte, das sie solchs mit Rechte noch in andere wege nicht vornehmen oder andern gestatten wolle.

Ehe sie nhu solche gelubde gethan seij sie gefengcklich enthalten biss vngevarlich vmb Martini (um den 11. November), da sie entledigt. Vnder des hab das Capittel ihr hauss zu Aschaffenburgck geoffnet, als was darijnne vnd was sie sunst zu Meinz beij sich gehabt zu sich genohmen, welches sich vngefarlich biss in dije etlich 10 tausent gulden erstreckt. 
Haben ihr nichts fernners gelassen, dan dije brieve vnd siegel vber ijhre Zinse vnd Renthe dije sije zu Halle (,) Leijpzig vnd Normbergck hat dije sie auch von ijhrem erbe vnd angefellen so sije zu Franckfurt gehabt vnnd noch zum teijl newlich ledig worden, erkaufft, welches sich vngeverlich in dije etlich tausent gulden heuptsum(m)a erstreckt sambt dem was ihr vnser gnedigster herre der Cardinal etc. seliger Nemlich 200 gulden zu Opfergelde gegeben, welch gelt sie sampt anderm also zu rhate gehalten vnd auffgesummet hette.

$\left(26^{r}\right)$ Als sie vmb dije stuck des so ihr genohmen gefragt hat sie bericht, das sije gehapt habe:

400 marck sijlbers an allerleij sijlbern vnd vorgulten schweren bechern vnnd Trinckgeschirr, ist in Sum(m)a vber $5000 \mathrm{f}$ (loren) wirdig geacht.

15 pfundt goldes an ketten vnnd allerleij Cleijnothern ausserhalb der edelgesteijne.

Item 1 Perlen Rock den Amanden der Seijdesticker gemacht vnd davon gueten bericht weijss.

Gulden vnd Seijdengewanth so noch vnvermächt vnd vngeverlich in die 1500 gulden wirdig.

90 Gurthel gross vnd kleijn darunder das beste vff 1200 gulden vnd noch eijns vff 700 gulden geacht.

Item etliche Pater noster vnd neheringe gulden vnd sijlbern.

Allen Haussrhat den sie in grosser anzahl in dem Newenhause zu Aschaffenburg gehabt vnd von dem ijhrem gezeuget, alleijne etliche geringe Bettlin vnd Leijnewanth, das sie ihr wider zuwerffen wolten.

32 Rocke dorober noch 10 die sie ihr widdergegeben.

Ite(m) vngefarlich 300 gulden ringe mit Edelgesteijnen.

Etliche viel geschmuckter Paneth (?) mit Cleijnothern geziert.

$\left(26^{v}\right)$ It(e)m 12 stuck weijns ijm hause zu Aschaffenburg davon sie zweij stuck in ein ander hauss gezogen habe ihr dije widder zuzustellen Aber doch wider in ihr hauss zu dem andern weijn bracht haben solten in meijnung vnd besorg das der zu guethe vnd das sije ihr 2 andere stuck dije geringer davor zu ihrer notturfft vorschaffen wolten.

$\mathrm{Zu}$ dieser vrfride hat sije zu Burgen setzen mussen Ern Johan Frisch ijhrem schwager, den Keller zu Meinz (,) Doctor Wijlhelmen zum Vogelsange (,) Adam Reijnmeijster vnd ijren Bruder (Johann Strauss) den Schultessen zu Awem (Auheim).

Vnd was ihr das Capittel also genohmen haben sie vnder sich vnd sagen da sie diesen ijtzigen newen Erzbischoff (Sebastian von Heusenstamm, 
1545-1555) erwelet, das sie ijhnen clerlich vorbehalten, mit ihr ijhres gefallens zuhandeln darijnne ijhnen der Newe Bischoff nichts zusagen haben solte.

Aber die Briever vnd Siegel sso ihr bleijben sollen, vnd davon oben meldung geschiedt hat das Capittel hinder den Rhat zu Meinz hinderlegt, vnd der Erzbischoff hat dije schlussel darzu beij sich, das sie derselbigen nicht mechtig.

Sie ist wol mechtig ab vnd zu zuziehen wohin sie wil. Aber das Capittel wil dannoch sie solle beij ijhnen zu Meinz sein vnd bleijben vnd sich andersswoe nicht zubesetzen haben. Vnd wiewol ihr solchs durch $\left(27^{r}\right)$ ijhren gethanen Vrfriden vnd Burgschafft nicht benohmen, so ist doch wol zuerachten, weijl sie ihrer briefe vnd siegel, so ihr bleijben sollen nicht mechtig, das sie dadurch gedrungen zu Meinz zubleijben.

Sije Bitt aber vndertheniglich, das vnser gnedigster Herre der Erzbischoff zu Magdeburgck etc. (Johann Albrecht von Brandenburg, 15451550) ihr von dieser ihrer gethanen vorpflichtung vnd gesezten Burgschafft helffen vnd sie mit gnaden schutzen vnd hanthaben vnd das sochs nicht vff ire bijtte vnd ansuchen sonder seiner $f$. $g$. als vor sich selbst aus gnaden thun wolte den ir auch zuclagen verbotten vnd hart eingebunden kein clage nirgent vorzubringen.

Sie zeigt auch an, das ihr vnser gnedigster Herre der Cardinal etc. offtmals gesagt, wan sie nach seiner Churf. g. tode anfechtung haben wurde, das sie zu vnserm gnadigsten Herren dem Erzbischove etc. Marggrafe Johan Albrechten (1499-1550) vnd auch sonderlich Marggrave Albrechten zu Anspach (von Brandenburg-Ansbach, 1490-1568) zuflucht haben solte, die wurden sie mit schutz vnd handhabung nit verlassen. Bijth an vnserm gnedigsten herren dem Erwelten zuerkunden, ob sie auch sein f. g. darumb schreijben mochte, das ijhr dasselbige zu keijner gefehr gereiche.

Bericht fernner, das sie an ijhrem sijlbergeschirre ijhr lebtage nichts aus der sijlber Chammer bekommen, sonder wisse ein ijdes von weme sie es bekommen anzuzeigen vnd seij ihr nicht alleijne von vnsernn gnedigsten herren sonder anderer meher hern geschenckt $\left(27^{v}\right)$ wie sie dan von eijner Kethen gesagt die ihr herzog heinrich zu Braunschweigk (-Wolfenbüttel, 1489-1568) geschenckt gewest sein solle.

So hab ihr auch sein churf. g. nie keijn mahl zugleich 1000 gulden werth gegeben sonder je zuzeiten 1 ringlein, dan ein gurthel vnd also einzeln gegeben vnd seij solchs sein eijgen freij guth gewest nicht auss den 
Stiefften oder derselben angefellen herkommen. Das sije also mit dem was ihr zu schulden keine vrsach geben, hab sich auch erbothen woe das Capittel echts schulden anzuzeigen wisse, das sie vorvrsacht, das sie dieselben schulde von dem ihren bezalen wolle.

Sie hab auch das hauss zu Aschaffenburg von dem ijhren erbawen lassen vnd sein die 2200 guldenn welche von dem Grunen hofe zu Halle ijhr erlegt darzu gebraucht worden, so hab sije der frohne halber vnd kost meijnem guet(igen) herren vorgleichung gemacht, das sije derhalben nijemant echts schuldig oder zuthun seij etc.

(28) Der Cleijnoth Ornata vnd anders halben bericht sije:

Das alle Cleinoth vnd Ornata, so ijhm Thirgarten zu Aschaffenburg (Heiliggrabkirche der Beginen) biss in den todlichen abgangck gewest, noch alle furhanden, die sich vngevarlich in die 8000 gulden erstrecken.

Von voranderung der wapen das Meinzische wapen an Magdeburgisch oder Halberstadisch wapen stadt gesezt sein solten davon wisse sije nicht hab auch noch zu lezst daraus gessen vnd die Magdeburgisch vnd Halberstadisch wapen darauff gesehen.

So seint auch die furnembsten Stiefft, Closter vnd Hewser ijm Stiefft Meinz mit dem was (aus dem Hallenser Stift) herrauss khommen staffirt vnd vorsehen, das auch alleijne in das Stiefft zu Aschaffenburg ober die $18000 \mathrm{f}$ (loren) khom(m)en.

Dije Bucher mit den schonen frembden munzen welche funff gewest, 2 Bucher von gulden vnd 3 bucher von sijlbern munz(en) hab auch das Capittel zu sich genom(m)en. Desgleichen die Tapezereij so allenthalb vorhanden gewest.

Sije hab auch gehabt viellerleij eingemacht dingck von specereij vnd anderen vngewerlich auff 500 gulden wirdig. Das hab der nawe Erzbischoff vom (Aschaffenburger) Schloss genohm(m)en vnd sol es in die Apotecken verkaufft haben.

$\left(28^{v}\right)$ Bericht weijther was von Silbergeschirr oder andern vorkaufft, seij alles vff den Reichstaegen gescheen vnd auffgangen.

Zeigt auch an, das vnser gnedigster herre seliger gedechtnus einssmals zugleich Einmalhundert thausent gulden bekhommen, vnd (-) das jerlich 7000 thaler vom Konige Pension gehabt, darauff sein churf. g. fur zweien jharen vngeverlich 21000 gulden entpfangen vnd sich auff dreij jhar hat bezahlen lassenn etc. 
Sije hat auch angezeigt, das das Capittel vorgewenth, vnser guet(iger) herre der Cardinal etc. loblicher gedechtnus, hette kein Testament machen konnen, weije s. churf. g. alle ihre schulde zuvor nicht bezalt.

Sije hat sich auch weijther erbathen, da sein $\mathrm{f}$. g. fernnern bericht von ihr haben wolte were sie bereijt von allem dem das sije wuste Seinen f. g. vnd derselben beijden Erz vnd Stiefften zum besten eijgentliche anzeigung zuthuen, alleijne das sije auch gnedigs schuzes vnd handthabung sich zutrosten haben beij gleich vnd recht erhalten werden mochte.

Sije zeigt auch an, das sie zu Calbe ijm grossen Lanttag (Januar-Februar 1541) vnserm guet. hern dem Cardinal hochloblicher gedechnus 1000 thaler vorgestreckt die stehen ijhr noch aussen hat nije daruff sonderlich gedrungen etc.

Desgleichen hernach hab sije S. churf. g. $500 \mathrm{fl}$. auch hernach vorgestrackt stehen ijhr auch aussen.

$\left(29^{r}\right)$ Sie sagt auch, das alle Tapezereij wie die von Halle sampt dem Haussraht gefurth nach absterben vnsers g. hern vorhanden gewest vnd noch sein mussen.

Bittet ganz demutig, das ijhr dieser ijhr bericht zu guthe gehalten vnd ijhr daraus nit weither beschwerung erwachsse. Dan sije denselbigen vnserm gnedigsten hern vnd den beijden S. f. g. Stiefften Magdeburg vnd Halberstat zu guthe gethan. 